\section{Fédéralisme et Disparités Culturelles en Belgique}

\section{Par Sylvie Tremblay*}

* Sylvie Tremblay a obtenu un baccalauréat en science politique à l'Université Laval en 2003. Elle poursuit actuellement une maîtrise en étude de l'environnement à l'Université York de Toronto.

\section{Abstract}

This article examines the effects of Belgian federalism on its linguistic minorities, specifically the French, Flemish and Flanders. The author beings by posing the question of whether Belgian federalism has resolved the cohabitation of its different linguistic groups. He then outlines the historical background of the difficulties between the groups, as well as provides a description of the federal structures that have been adopted to improve these difficulties. An important aspect of the paper is its analysis of the outcome of these political structures on the interactions between the minorities. It finally concludes that federalism in Belgium has failed to integrate the different communities, as it has not fostered national unity. Some indicators predict that there is a threat of the collapse of the nation, and that this is a serious cause for attention.

\section{Introduction}

Deux voies mènent à un État fédéral : l'union d'entités préexistantes ou la division d'un État unitaire en différentes entités politiques nouvelles. C'est cette dernière option qui définit le cheminement de la Belgique fédérale que nous connaissons aujourd'hui. Dans la plupart des fédérations, le recours à un régime gouvernemental à plusieurs niveaux est justifié par une diversité ethnique, culturelle ou linguistique au sein de l'état qui implique un obstacle majeur à la gouverne. La Belgique ne fait pas exception à cette règle. On y constate une opposition de longue date entre les deux groupes linguistiques dominants : les Wallons et les Flamands. Le régime fédéral tel que construit par ce pays revêt des couleurs particulières qui veulent s'accorder avec sa composition démographique. La question qui se pose est la suivante: le fédéralisme tel qu'appliqué en Belgique a t-il résolu les problèmes de cohabitation des différents groupes linguistiques qui la compose?
Pour arriver à une réponse, il faudra s'attarder d'abord à l'évolution historique des difficultés. La description de l'organisation du fédéralisme belge sera la deuxième étape de l'analyse : il s'agira de dresser un portrait global de la structure fédérale, des entités qui la compose et des compétences respectives de ces dernières. Certaines caractéristiques du régime seront expliquées : la polarisation qu'il a créée, la situation des minorités que l'on peut y reconnaître, les politiques linguistiques et culturelles qui ont y été développées. À l'issue de cette réflexion, nous serons en mesure de donner certains éléments de réponse à notre interrogation qui se révèle d'une complexité non-négligeable, tout comme son sujet d'étude : le fédéralisme belge. Ce que nous découvrirons, c'est que le but du fédéralisme a été de faire place à la diversité que l'on retrouve sur le territoire belge. Toutefois, ce faisant, il a omis d'assurer un maintien suffisant de l'unité nécessaire à la cohésion du pays. Ainsi, il n'aura, à la fin, pas réussi à établir une formule efficace pour assurer la pérennité de la Belgique. Du moins, si le pays n'est pas à la veille d'un écroulement, il y a raison de s'inquiéter.

\section{La Belgique unitaire et ses premiers pas vers la décentralisation}

La Belgique a été créée en 1830 suite à une révolution menée par la bourgeoisie francophone des Pays-Bas qui a entraîné avec elle une partie de la petite bourgeoisie néerlandaise. Les deux groupes craignaient la marginalisation linguistique, pour les Francophones, et religieuse, pour les Néerlandais catholiques, qui aurait pu résulter des politiques uniformisantes de Guillaume D'Orange (Lecours : 2002, p. 141). C'est le français qui a alors été choisi comme langue de fonctionnement dans les domaines politique, économique et d'éducation pour le nouvel État. Il en fut ainsi puisque ce furent les francophones qui instiguèrent la rébellion et que leur langue représentait un prestige plus grand que le néerlandais, perçu comme un langage rustre.

La dominance du français s'est bientôt révélée synonyme d'assimilation pour les Flamands. Alors que l'on comptait très peu de Francophones bilingues, les Flamands devaient maîtriser le français pour grimper l'échelle sociale. Les Wallons se montrèrent intraitables quant à l'utilisation du néerlandais dans le secteur public. Un événement important dans l'histoire illustrant ce problème fut la confusion que créa l'unilinguisme français pendant la Première Guerre Mondiale. Effectivement, les soldats flamands, qui ne comprenaient rien aux ordres de leurs supérieurs, y périrent en nombre important. À la lumière de ces explications, on peut comprendre la polarisation du mouvement flamand né 
quelques décennies plutôt à la fin du XIX siècle. (Morelli : 1995) Par la suite, différents facteurs ont résulté de l'élargissement de la conscientisation flamande et ont fait avancer le mouvement. Notamment, l'universalisation du scrutin (autrefois réservé à la classe privilégiée dont les qualifications académiques étaient exigées) et l'établissement de politiques linguistiques encourageantes (1920-30) ont été des mesures cruciales à l'évolution du mouvement. De plus, l'infiltration grandissante du français en région flamande, particulièrement dans les environs de Bruxelles, a motivé des doléances du côté flamand. Il se produisait la chose suivante : comme la législation voulait que les communes qui dépassaient le seuil de $50 \%$ de francophones changent de langue d'usage, on vit la frontière linguistique se déplacer lentement vers le nord. Les Flamands décrivaient cette progression géographique des francophones comme une «tache d'huile»et cela leur causait beaucoup d'inquiétude. Ce sont ces conditions qui ont mené à un double unilinguisme régional, politique dont nous reparlerons plus bas. Quoi qu'il en soit, ce fut le début du développement d'un véritable nationalisme flamand.

Karmis et Gagnon montrent comment a émergé le mouvement homologue wallon: «Davantage réactif, le mouvement wallon ne vise à l'origine que la revitalisation de la culture et des dialectes wallons. Il se politise face aux lois linguistiques, perçues comme la menace d'une mainmise flamande sur l'État.» (Karmis et Gagnon: 1996, p. 441) En outre, le mouvement wallon reste faible jusque qu'à la moitié du $20^{\mathrm{e}}$ siècle, période où il se mobilise autour d'enjeux économiques. La Wallonie, traditionnellement plus riche en raison de son activité minière, vit son industrie décliner au cours du $20^{\mathrm{e}}$ siècle. La richesse était leur force, leur sécurité, la base de leur contrôle sur les Flamands plus nombreux statistiquement. Ainsi, devant la peur de se voir dominer économiquement par ses voisins, le mouvement wallon se solidifia. La prise de conscience du côté wallon est davantage un régionalisme qu'un nationalisme parce que ses bases furent plutôt économiques que culturelles.

Les deux mouvements s'accentueront dans les années 1960 et en conséquence, une nouvelle radicalisation des politiques linguistiques éliminera les éléments de bilinguisme qui subsistaient à l'échelle nationale. Bientôt, les faits devinrent indéniables : la Belgique était aux prises avec un régime qui ne convenait plus à sa réalité démographique, linguistique et culturelle. En 1970, le Premier-ministre Eyskens fait une déclaration et reconnaît que l'état unitaire est dépassé par les faits et que l'on doit rénover les structures de l'État.

\section{Organisation du fédéralisme belge}

«Dépassé dans les faits, l'État unitaire décentralisé établi par la Constitution belge de 1831 allait entamer sa métamorphose fédérale en 1970.» (Wilde d'Estmael : 1996, p. 93) Les transformations qui ont fait de la Belgique un État fédéral se sont faites par étapes. Les principales modifications constitutionnelles se sont produites en 1970, 1980, 1983, 1988-89 et 1993. C'est à partir de cette dernière année que la Belgique s'est attribuée à ellemême le titre de fédération. Selon l'avis de certains auteurs, ce fédéralisme visait la pacification sans vraiment avoir de «vision d'ensemble» (Karmis et Gagnon : 1996, p. 456). On organisa un fédéralisme de dévolution, ce qui est aisément explicable par l'histoire unitaire du pays. Il aurait été surprenant de voir les Belges se doter d'un régime centralisateur. Le danger qui les menaçait et dont on ne se méfia sûrement pas suffisamment était le cloisonnement des entités fédérées. Les prochains paragraphes auront pour but de montrer comment la Belgique est fractionnée de par le fonctionnement même du fédéralisme qui la structure.

\section{Les entités fédérées et leurs compétences}

Le fédéralisme belge est plutôt complexe de par la superposition de ses entités fédérées. Effectivement, les Flamands ayant revendiqué la création d'une communauté culturelle incluant Bruxelles et les Wallons désirant la formation de régions établies selon des considérations économiques, on a opté pour une combinaison quelque peu inusitée. La Belgique a été divisée en trois communautés : Flamande, Française et Germanophone. Ce sont des entités linguistiquement déterminées comprenant respectivement 6 millions, 4 millions et 60000 habitants. Les communautés ont pour mandat de légiférer sur ce qui se rapporte à la personne : enseignement, culture, etc. En complément aux communautés, on a eu recours à une division régionale du pays basée sur des critères territoriaux. En ont résulté les régions flamande au nord, wallonne au sud et Bruxelles-Capitale au centre. (Wilde d'Estmael (de): 1996, p. 94) On comprend que la région et la communauté flamande sont les seules à coïncider géographiquement, ce qui a encouragé ces dernières à joindre leurs compétences dans une seule institution législative. Les pouvoir qui ont été réservés au niveau du gouvernement central sont uniquement ceux qui relèvent de l'unité socio-économique et territoriale, soit : la monnaie, les finances, la sécurité sociale, la justice et la défense (Jaumain : 1997, p. 3) Les deux niveaux de gouvernement (fédéral et régional) ont des gouvernements autonomes dotés d'un corps législatif représentatif. Ce qu'il faut retenir, c'est que le gouvernement central n'a aucun contrôle sur toutes les décisions d'ordre culturel ou social et ne peut 
donc pas intervenir dans ces champs qui sont déterminants dans le maintien d'une certaine unité au pays.

L'organisation institutionnelle fédérale en Belgique est déterminée par une asymétrie frappante. Les différentes philosophies des collectivités expliquent cette réalité. Alors qu'au Nord, la communauté et la région se sont jointes administrativement, au sud, il en est tout autrement. Le pouvoir de la communauté française est limité en comparaison à celui de ses voisines. Il en est ainsi parce que la région de Bruxelles-Capitale est autonome par rapport à la Flandre (cela prive la Wallonie d'un poids politique considérable) et parce que la communauté française à eu recours à l'aide financière des régions flamande et bruxelloise (elle s'est retrouvée en situation de dépendance par rapport aux autres.) La considération de cette réalité, juxtaposée à d'autres complications administratives, permet un début de compréhension de l'asymétrie mentionnée ci-dessus. Pour ne nommer qu'un dernier exemple d'asymétrie, la communauté germanophone a exprimé elle aussi le désir de contrôler sa partie de territoire régional. De façon concise : «les trois Communautés et les trois Régions ne se trouvent pas sur un pied d'égalité, puisqu'elles entretiennent des relations plus ou moins privilégiées avec les communautés ${ }^{1}$ flamande et francophone.» (Delgrange : 1995, p. 1170) Dans un État fédéral où la méfiance envers les autres groupes culturels fait partie de l'histoire, il est évident que cette asymétrie représente un potentiel de jalousie, de conflit et de fracture éventuelle.

\section{La polarisation}

La façon dont la Belgique est devenue un État fédéral donne un premier élément d'explication à la polarisation qu'on y décèle aisément. On se rappelle que la Belgique a eu recours à la dévolution. Plusieurs auteurs sont en accord pour dire que le caractère dévolutif de la fédéralisation belge est crucial dans la compréhension du problème : «On imagine mal un passage de l'unitarisme au fédéralisme affirmant la prépondérance d'une identité panbelge. Toutefois, du fait d'un manque de préoccupation pour l'unité, les réformes belges pèchent par excès inverse et tendent vers une institutionnalisation de la fragmentation.» (Karmis et Gagnon : 1996, p. 457)

Pour compliquer la donne, Xavier Delgrange ajoute un élément de fragmentation du peuple belge. À la ligne de fracture linguistique

${ }^{1}$ Pour l'auteur, le mot communauté écrit avec une minuscule réfère à la collectivité et non à l'entité fédérée.
(Flamands et Francophones), il additionne la composante idéologique (les catholiques sont majoritaires en Flandre et les laïques, en Walonnie). L'appartenance des habitants à l'un ou l'autre des piliers idéologiques a été d'une grande importance lors de débats qui ont eu lieu en relation à des thèmes controversés comme l'enseignement et l'avortement. (Delgrange : 1995, p. 1171) Ce facteur a toujours été présent mais il semblerait qu'on avait trouvé, au sein de la Belgique unitaire, un certain équilibre. Le fédéralisme a, semblerait-il, exacerbé la division idéologique des Belges.

La Belgique donne une impression nette de fragmentation en son sein. Sur ce sujet, André Lecours résume en donnant trois éléments d'explication. Il attribue premièrement cette impression à la complexité de l'organisation fédérale et au degré important d'autonomie des entités fédérées par rapport au centre. L'exemple ultime de cette autonomie est assurément le pouvoir de négociation des communautés et des régions au niveau supra-national. Deuxièmement, l'auteur a remarqué la tendance centrifuge des institutions belges depuis 1970. Selon lui, on s'approche de plus en plus d'un régime confédératif. Troisièmement, la polarisation du système de partis est significative. Effectivement, l'absence de partis nationaux, d'une part, et le fait que les assises des partis soient linguistiques d'autre part, en disent beaucoup sur la situation. Cette expression concrète de la division de la Belgique est très significative et s'est manifestée pour la première fois dans les années 1960. Ce fut en 1965 que les partis communautaires ont commencé à prendre plus de place aux élections fédérales. Cela a remis en question les stratégies des grands partis nationaux (partis Social-Chrétien, Socialiste et Libéral). Ces derniers ont dû adapter leur plate-forme aux nouvelles exigences nationalistes des communautés. Dans cette période turbulente, des conflits internes ont éclaté au sein même des partis qui se sont divisés en 1965 pour les Sociaux-chrétiens, et en 1966, pour les Socialistes. Même les Libéraux, parti le plus fort et dont les liens avec le centre étaient les plus forts, ont vu leur structure ébranlée à partir de 1968. (Lecours : 2002, p. 144) Une nouvelle dynamique politique s'est installée en Belgique, une dynamique de fragmentation partisane. Selon Lecours, c'est ce changement qui a été catalyseur de nouvelles revendications pour le démantèlement de la Belgique unitaire. Il ajoute :

The existence of linguistic wings and autonomist strands produced a political dynamic whereby sections of language groups within traditional parties sought to create new institutions to neutralize community parties without jeopardizing their political-electoral fortune. It introduced a pattern of élite 
competition that saw the relationship between traditional parties become heavily conditioned by a nationalist/regionalist perspective. (Lecours: 2002, p. 145)

Pour conclure sur le sujet de la polarisation, il faut garder en tête l'importance d'un équilibre entre la diversité et l'unité dans une fédération. Les éléments polarisateurs qui ont été énumérés dans cette partie de l'analyse montrent comment le fédéralisme tel qu'appliqué en Belgique ne respecte pas cet équilibre. À défaut de corriger cette déviation, la Belgique risque l'éclatement.

\section{Les minorités}

Les Belges ont eut recours au fédéralisme pour satisfaire leurs collectivités linguistiques qui n'arrivaient pas à s'entendre dans un État unitaire. Toutefois, ce faisant, ils ont créé de nouvelles minorités présentant des exigences particulières. Commençons par le début : on sait que les Wallons représentent une minorité en termes de nombre. Cependant, ils ont dominé la Flandre linguistiquement pendant longtemps. Ainsi, on pourrait dire, comme le suggère Delgrange, que les Flamands sont, ou ont été, une minorité psychologique. (Delgrange : 195, p. 1162) Plus récemment, les Wallons se sont retrouvés face à un mouvement flamand se fortifiant, ce qui a influé négativement sur leur assurance et leur pouvoir en tant que majorité psychologique. Ils ont dû se rendre à l'évidence et reconnaître leur statut minoritaire. Le fédéralisme a apporté des solutions à ce dilemme par la décentralisation que ses différentes étapes ont impliqué. Maintenant, voyons comment les nouveaux dilemmes se présentent.

À cause de leur importance moindre en termes géographiques et démographiques, on pourrait dire que la Région de Bruxelles-Capitale et la Communauté germanophone sont des minorités institutionnelles. De fait, elles sont traitées différemment des autres entités. L'asymétrie qui a été décrite précédemment relativement aux pouvoirs et aux compétences régionales/communautaires constitue la base légale de la différenciation du traitement réservé aux minorités institutionnelles. Ces minorités se voient souvent accorder une importance moindre que leurs régions ou communautés voisines :

Fondamentalement bipolaire, puisque reposant sur les communautés flamande et française qui font peu à peu place aux nations flamande et wallonne, [le fédéralisme belge] privilégie les entités qui sont les émanations directes de ces deux nations, la Communauté flamande d'un côté, la Région wallonne et dans une moindre mesure la Communauté française, de l'autre. (Delgrange : 1995, p. 1172)

On comprend que Bruxelles-Capitale et la Communauté germanophone n'entrent pas dans ce cadre : la première est bilingue, mais peuplé à $90 \%$ de francophones et l'autre parle une langue différente. Une tension inévitable est née de cette asymétrie et constitue un potentiel de frustration.

Dans un autre ordre d'idées, en plus de mettre en lumière les minorités entre les différentes entités fédérées, le régime fédéral belge a provoqué l'apparition de minorités à l'intérieur même des communautés et régions. Historiquement, les conflits qui ont eu lieu ont rendu nécessaire l'établissement de mesures de protection des minorités laïque en Flandre et catholique en Wallonie. Ce sont des minorités idéologiques. La protection de ces minorités est compromise par l'impuissance du gouvernement central à leur assurer une reconnaissance. On peut, de surcroît, imaginer les tensions dues au double unilinguisme, et ce, particulièrement dans la région Bruxelles-Capitale. Comme les minorités idéologiques, les minorités linguistiques risquent la discrimination dans le régime fédéral tel qu'on la comprend à l'heure actuelle.

\section{Politiques linguistiques et culturelles}

Afin de protéger les différentes minorités présentes sur le territoire belge, plusieurs mesures ont été mises en place au cours des décennies et demeurent sous le nouveau régime fédéral. La première à laquelle on pense est l'unilinguisme. Cette mesure peut paraître excessive, surtout dans un pays où le bilinguisme a déjà été favorisé dans certains secteurs où les besoins le justifiaient (administration centrale, service public, par exemple).

L'unilinguisme s'est installé progressivement dans la première moitié du $20^{\mathrm{e}}$ siècle. À ce moment, ce sont les francophones qui se sont opposés à la généralisation du bilinguisme. Ainsi, afin d'assurer l'égalité des langues, chacun a opté pour l'unilinguisme double et rigide. (Domenicelli : 1999, p. 44) Si une seule chose reste positive dans cette situation, c'est que les deux communautés s'entendent actuellement sur les politiques linguistiques en place. Ceci dit, Domenicelli précise que si on poursuivait notre observation sur ce sujet, on verrait que s'il y a entente au sujet de l'unilinguisme, l'arbitrage au niveau des matières soumises à la réglementation crée des insatisfactions. Brièvement, les mécontentements sont dus à des différences d'interprétation: les Francophones comprendraient que la législation signifie que nul ne se voit contraint de maîtriser les deux langues et les Flamands, eux, seraient d'avis qu'il est 
interdit d'utiliser une autre langue que celle parlée sur le territoire. (Domenicelli : 1999, p. 46) On oppose la liberté à l'interdiction. En outre, un article de la Constitution belge prévoit l'assurance d'une liberté linguistique (art. 30) mais cet article a suscité moult débats. L'enjeu soulevé fut le maintien des disparités que causerait une telle liberté. La défense des langues dans les différentes communautés serait rendue impossible. Ce débat peut être réduit à deux concepts : le droit collectif que réclament les Flamands de protéger leur langue contre le droit individuel revendiqué par les Francophones d'utiliser sa propre langue si la situation le permet. (Domenicelli : 1999, p. 28) Cette longue explication cherche à montrer que les deux collectivités diffèrent dans leur interprétation de la législation et que cela leur pose clairement problème.

En ce qui a trait aux minorités à l'intérieur des entités fédérées, elles ne sont tout simplement pas considérées par la Constitution qui stipule que les communautés sont homogènes linguistiquement (à l'exception du territoire qui correspond à la région Bruxelles-Capitale qui est bilingue). (Delgrange : 1995, p. 1178) Cela remet toute la responsabilité de la protection de ces minorités dans les mains des communautés qui exigent généralement, d'ailleurs, une homogénéité. Les raisons à cette politique ont été données dans le paragraphe précédent. C'est surtout la Communauté flamande qui a exprimé une préoccupation pour l'application stricte de ce principe. Parce que le fédéralisme est organisé de sorte à laisser libre les entités fédérées en matière de politique linguistique interne, cela leur est permis. Encore une fois, il semble clair qu'une telle situation pose un grand risque d'injustice ou de mécontentement de la part des minorités linguistiques ou idéologiques qui se trouvent marginalisées.

\section{Analyse : quel est le véritable rôle du fédéralisme dans le maintien de l'unité?}

Après avoir dressé le portrait global du fédéralisme belge, on en revient à la question de départ, à savoir: le fédéralisme tiendra-t-il son pari initial et réussira-t-il à réconcilier les collectivités qui composent la Belgique? Cette interrogation suscite des réactions diverses chez les auteurs qui offrent certains éléments de réponse.

\section{L'écart idéologique entre la population et ses élites}

En premier lieu, André Lecours énonce un paradoxe. À la conception populaire qui dit que l'état belge est irrémédiablement fragmenté, il oppose les données empiriques qui montrent une population attachée à son État. Il précise : «[the empirical investigations] reveal that the fragmentation of politics and institutions in Belgium has not been accompanied, or preceded, by an equally important fragmentation of identities.» (Lecours: 2002, p. 139) Cela suggère que la population n'appuie pas ses élites. L'auteur cité ici prend un point de vue intéressant en divisant la dynamique des mouvements nationalistes/régionalistes et la population qu'ils prétendent représenter. Il propose de réviser notre perception de la situation belge en diminuant la quantité de pouvoir que l'on accorde aux identités nationales diverses. Empiriquement, il argumente que les mouvements communautaires n'ont pas réussi à avoir l'appui de la population générale avant la fin du $19^{\mathrm{e}}$ siècle pour les Flamands et les années 1960 pour les Wallons. Jusqu'à ces époques, ils n'arrivaient à rien faire bouger dans la structure unitaire. Ce n'est que l'indifférence du peuple qui a pu retarder ainsi le processus. De plus, on peut douter que la division linguistique des grands partis ait vraiment résulté de la demande populaire. Il est plus probable que la menace des petits partis, en 1965, ait provoqué une réaction exagérée si l'on tient compte de l'opinion réelle des belges à l'époque. Le résultat fut une sur-représentation des intérêts communautaires par les partis et un accroissement des tensions. Lecours propose un appui empirique supplémentaire à son argumentation : il utilise des résultats de sondages réalisés entre 1975 et 1995 . Ceux-ci montrent que l'attachement de la population à la Belgique a grandi de façon inversement proportionnelle au développement de la fédération ${ }^{2}$. (Lecours : 2002, p. 141) Pour cet auteur, l'écart idéologique entre la population et ses élites communautaires constitue l'explication au maintien de l'unité belge jusqu'à présent. En conséquence, une conscientisation par rapport à cette réalité permettrait un avenir plus certain pour la Belgique.

\section{Un système complexe dont la pertinence reste à établir}

Pour un autre auteur, Xavier Delgrange, le problème de la Belgique relève principalement de la complexité de ses institutions. Dans le but de satisfaire les demandes des différentes collectivités, la Belgique a installé sur son territoire un système de superposition comprenant six entités distinctes. On aurait pu s'attendre à ce que cela amène un certain degré de satisfaction. Il s'avère que ce n'est pas le cas pour tous. Dans un premier ordre d'idées, la

\footnotetext{
${ }^{2}$ Chez les Wallons, l'auteur note un pourcentage de gens s'identifiant d'abord à l'État central correspondant à 58\% en 1986 et à 69\% en 1992. En Flandre, 44\% des individus se disaient davantage attachés à la Belgique qu'à la région/communauté flamande pour laquelle on comptait un pourcentage de 33\% en 1986. (Lecours : 2002, p. 141)
} 
création des communautés a suscité des espoirs différents pour les Flamands, qui les voulaient territorialement déterminées, et les Francophones, qui envisageaient une appartenance linguistique. Évidemment, ce sont les Wallons qui se sont vus déçus par la tournure des évènements. Leur espoir d'avoir un mot à dire par rapport aux minorités francophones à l'extérieur de la Wallonie a été anéanti par Robert Colignon, Ministre-Président de la Région wallonne qui a clairement fait savoir qu' «un fédéralisme sain est territorial» ${ }^{3}$.

Dans un second ordre d'idées, il faut revenir aux propos qui ont été tenus dans les pages précédentes au sujet des minorités institutionnelles. Celles-ci se retrouvent souvent éclipsées dans un système dont la logique est nettement bipolaire. Bruxelles-Capitale et la Communauté germanophone sont même considérées différemment par la constitution : elles sont privées de certaines compétences attribuées aux autres entités homologues. Delgrange nous apprend que ces deux collectivités, contrairement à leurs pairs, n'ont aucune autonomie en ce qui concerne «certains aspects des élections, de la composition et du mode de fonctionnement des Conseils et des Gouvernements» (Delgrange : 1995, p. 1186) D'autres caractéristiques techniques distinguent les différentes entités. La somme de ces caractéristiques rend la situation plutôt précaire pour les minorités institutionnelles, particulièrement Bruxelles-Capitale, qui se trouvent dépendantes des ententes contractées entre les deux collectivités dominantes, soit les Flamands et les Wallons. La conclusion est la suivante : le fédéralisme a amené avec lui de nouvelles complications qui empirent peut-être le problème de cohabitation au lieu de l'améliorer.

\section{Un excès de particularismes}

De leur côté, Karmis et Gagnon attribuent les problèmes des belges aux excès de particularisme auxquels ils se sont adonnés. Selon eux, la fragmentation qui les afflige est due au manque de considération pour l'unité nationale. À trop considérer la diversité dans l'aménagement politique, ils n'ont rien mis en place pour assurer une cohésion. Nous avons déjà, dans ce travail, fait mention du cloisonnement qui caractérise le fédéralisme belge. Depuis la mise en œuvre de la constitution fédérale de 1993, ce cloisonnement s'est accentué suite à diverses controverses politiques. En Flandre, on a développé un ressentiment face à la Wallonie qui a traversé une période économique difficile. Sollicités par les Wallons

${ }^{3}$ Xavier Delgrange tire cet extrait de: Le Soir, 9 mars 1994, p. 3. (Delgrange : 2002, p. 1186) dans différents programmes de redressement économique, les Flamands on fait fi de la solidarité intercommunautaire et ont refusé de collaborer. S'en est ensuivi, dans les prochaines années, tout un débat sur la responsabilité économique des entités fédérées par rapport à leurs voisines. Toutefois, les Wallons ont eux aussi installé une distance politique entre leur Région et l'État central. Cela s'est produit grandement en réaction à l'attitude des Flamands. À la fin, ce que Karmis et Gagnon soulignent, c'est qu' «En Belgique, les réformateurs ont laissé s'exprimer l'asymétrie, mais dans une perspective de cloisonnement ne favorisant pas l'existence d'identités plurielles.» (Karmis et Gagnon : 1996, p. 463) Bien que, dans les dernières années, il semblerait que certains efforts aient été faits en faveur de l'unité (voir les débats sur la loyauté fédérale), il apparaît sans équivoque que la Belgique doit réaffirmer les priorités de son régime, qu'elle doit trouver une façon de favoriser la communication entre ses collectivités qui évoluent dans des directions opposées.

\section{La représentation des entités fédérées au niveau supra-national}

Plusieurs auteurs font l'analyse du fédéralisme belge dans le cadre plus large de l'Union Européenne. Il est intéressant de définir le nouveau rôle que peuvent jouer les collectivités belges dans une Europe des Régions. Cela remet en question tout le concept de l'État-Nation tel que connu jusqu'à maintenant sur le continent européen. En effet, si ce dernier devient inutile, on laisse la porte ouverte au développement de l'autonomie de chacune des entités qui composent les pays participant à l'Union Européenne.

Les entités fédérées en Belgique ont le contrôle des relations extérieures dans tous les domaines qui correspondent à leurs compétences. Normalement, ce pouvoir revient aux autorités fédérales. À la limite, certains États permettent aux entités fédérées de signer des traités internationaux, mais sous la surveillance du gouvernement central ${ }^{4}$. Ainsi, le fédéralisme belge est, sur ce point, complètement atypique. On peut analyser cette particularité de plusieurs façons. À première vue, il peut sembler évident que la participation des régions à la politique extérieure du pays accentuera l'autonomie et la fragmentation. Les entités fédérées de Belgique sont les seules à bénéficier d'un pouvoir d'influence si grand au sein de l'Union Européenne. D'un autre côté, les régions et communautés

\footnotetext{
${ }^{4}$ Le gouvernement central belge doit approuver les signatures de traités par les entités fédérées mais il semblerait que cela soit davantage une formalité qu'une
} véritable implication. 
qui défendent leur point de vue, souvent identique, au niveau supranational, réaliseront peut-être qu'elles partagent bon nombre de préoccupations. En fait, peut-être vont-elles être mieux solidaires dans un cadre élargi à l'Europe en entier. Françoise Massart-Piérard a réfléchi à cette interrogation et en arrive à une réponse plutôt nuancée. Elle reconnaît que les états fédéraux aux prises avec des tensions centrifuges portent souvent leurs espoirs vers le niveau de gouvernement supérieur. Ils souhaitent voir la dévolution des pouvoirs mener à une pacification sur le territoire national. Ce que l'auteure retient sur ce sujet, c'est que la logique se gâte quand on regarde les objectifs du Nord et du Sud de la Belgique. Les Flamands voient leur rôle au sein de l'Europe de façon «nationalitaire» et les Wallons, de façon «régionalitaire». (Massart-Piérard : 1999, p. 18) Bref, les Flamands acceptent de participer au fédéralisme que s'il leur apporte une souveraineté plus grande. Et la logique de fragmentation se perpétue...

Certains dirigeants belges affirment que la Belgique agit comme modèle pour l'Union-Européenne, que l'on peut voir en la Belgique l'Europe fédérale et décentralisée de demain. D'autres font toutefois remarquer la contradiction des orientations qui sont en cause: la régionalisation de la Belgique ne semble pas très compatible avec les buts unificateurs de l'Europe. Cette analyse, quoique intéressante, n'est plus tout à fait pertinente dans le cadre de ce travail qui cherche à évaluer la cohésion au niveau de l'État belge. Il est difficile d'établir avec certitude l'impact qu'aura la participation des entités belges dans les relations extérieures. Il semble que d'une façon ou d'une autre, le fédéralisme en place ne suffit pas à amener la cohésion nécessaire à l'établissement de bases identitaires solides au sein de la Belgique que nous connaissons.

\section{Conclusion}

L'histoire de la Belgique est assez éloquente quant à l'explication des évènements et circonstances qui ont mené au régime fédéraliste qui organise à présent ses institutions. Le chemin qu'ont emprunté les décideurs au moment des transformations constitutionnelles a mené vers une décentralisation peu commune. Le tout s'est effectué dans un but de pacification qui a rendu menaçant tout effort de maintien d'un pouvoir central fort. Cependant, certains craignent que la Belgique ait jeté le bébé avec l'eau du bain. Peut-être, au contraire, que la Belgique a évolué vers la seule option qui se présentait à elle et qu'une cohabitation entre les communautés qui la composent est tout simplement impossible. D'une façon ou d'une autre, le fédéralisme ne semble que passager, qu'une étape dans le processus d'une décentralisation qui s'arrêtera peut-être à la confédération, qui continuera peut-être jusqu'à une fragmentation totale.

Les seuls espoirs qu'offrent les spécialistes en ce qui a trait au maintien de l'unité belge viennent de deux sources : la reconsidération du rôle des élites communautaires et la participation des régions et communauté aux décisions de l'Union-Européenne. Si Lecours a raison et que la Belgique a plus d'appui au sein de sa population qu'il n'en paraît, il sera peut-être envisageable de réduire l'influence des élites nationalistes et régionalistes et de revenir à un équilibre entre la diversité et l'unité. Ainsi, on arrivera à un véritable fédéralisme. Autrement, il reste la possibilité d'un fédéralisme très décentralisé qui profite d'un niveau de gouvernement supra-national pour évacuer les tensions qui l'affaiblissent à l'interne. Si les champs dans lesquels les communautés peuvent négocier à l'international restent limités, peut-être que les Belges pourront rester unis sous un régime fédéral. Pour terminer, le fédéralisme peut difficilement crier victoire en Belgique. Les espoirs qu'on nourrissait en 1993 ne sont sûrement pas éteints mais si on en arrive à une cohabitation pacifique en Belgique, ce ne sera vraisemblablement pas sous un régime fédéral traditionnel. Il faudra en dessiner un modèle beaucoup plus décentralisé qu'à l'habitude. D

\section{Bibliographie}

DELGRANGE, Xavier. «Le fédéralisme belge: la protection des minorités linguistiques et idéologiques», Revue du Droit Public et de la Science Politique en France et à l'Étranger, 1995, pp. 1157-1202.

DOMENICELLI, Luisa. Constitution et regime linguistique en Belgique et au Canada, Bruxelles, Bruylant, 1999, 152 p.

GORIELY, Georges, Monique Lambert et Jacques Lemaire. Affrontements et intolérances, Bruxelles, Éditions de l'Université De Bruxelles, 1995, 204 p.

JAUMAIN, Michel. «Some features of cultural policies applied in Belgium, particularly in the French-speaking community», Journal of Arts Management, Law, and Society, Fall 1997, pp. 205-226.

KARMIS, Dimitrios et Alain G. Gagnon. «Fédéralisme et identités collectives au Canada et en Belgique : des itinéraires différents, une fragmentation similaire», Revue Canadienne de Science Politique, vol. XXIX, no 3, 1996, pp. 435-468. 
LECOURS, André, «Solving the Belgian Paradox: Political-institutional fragmentation, national identity and nationalist/regionalist politics», Canadian Review of Studies in Nationalism, vol. XXIX, 2002, pp. 139-151.

MORELLI, Anne. Les grands mythes de l'histoire de Belgique, de Flandre et de Wallonie, Bruxelles, Édition Vie ouvrière, 1995, 312 p.

MASSART-PIÉRARD, Françoise. «Les identités fédérées de Belgique, acteurs décisionnels au sein de l'Union Européenne», Politique et Sociétés, vol. 18, no 1, 1999, pp. 3-40.
VANDEWEGHE, Willy. «Belgian Federalism and the Language Issue» dans A. Allain, M. Dupont et M. Hearn (Eds), Les fédéralismes, Presses Universitaires du Septentrion, 1996, pp. 99-107.

WILDE d'ESTMAEL (de), Tanguy. «L'idée fédérale en Belgique» dans A. Allain, M. Dupont et M. Hearn (Eds), Les fédéralismes, Presses Universitaires du Septentrion, 1996, pp. 93-97. 